\title{
El cerebro y la complejidad
}

Entrevista realizada en 1995 por Jean-François Dortier a Jean-Pierre Changeux *

Traducción del francés al español de

Luis Alfonso Paláu-Castaño

Universidad Nacional de Colombia, Medellín, Colombia

lapalau@une.net.co

- El cerebro humano es considerado con frecuencia como la estructura más compleja del universo. ¿Cómo puede un neurobiólogo especializado en cerebro afrontar esa complejidad?

Ante todo, hay que desprenderse de un uso de la noción de complejidad que serviría para cubrir nuestra ignorancia. No se puede utilizar ese término para justificar el hecho de que no sabríamos nada, que la complejidad escapa al entendimiento porque hay demasiados factores en juego.

Dicho esto, efectivamente el cerebro es una estructura de una extrema complejidad. Algunos datos son suficientes para mostrarlo. El número total de células nerviosas en el encéfalo es del orden de cien mil millones, cifra espectacular para un órgano de apenas 1,3 o 1,4 kg. Esas neuronas se reparten en algunos centenares de categorías. Cada neurona establece alrededor de diez mil contactos con otras células nerviosas.

Si uno se interesa ahora por el funcionamiento de las neuronas constata que cada una puede sintetizar y liberar muchos neurotransmisores; además, el abanico de los neurotransmisores liberados es susceptible de variar.

Si uno se atiene solamente a los criterios de número de células, número de conexiones, diversidad de los neurotransmisores... la combinatoria que de acá resulta le da al cerebro del hombre una organización única por su complejidad con respecto a todos los otros seres vivientes.

* Cómo citar: Paláu-Castaño, L. A. (2018). El cerebro y la complejidad. Entrevista realizada en 1995 por Jean-François Dortier a Jean-Pierre Changeux. Ciencias Sociales y Educación, 7(14), 225-230. DOI: https://doi.org/10.22395/csye. v7n14a12

Traducción retomada de: Dortier J.F. (dir.) (2012). El Cerebro y el Pensamiento. La Nueva edad de las ciencias cognitivas (pp. 95-101). Auxerre: Sciences Humaines éditions.

Agradecemos a la editorial francesa Sciences Humnaines autorizar la publicación en español de la entrevista al neurobiólogo, profesor honorario del Collège de France, Jean-Pierre Changeux. Nota del editor.

Recibido: 23 de agosto del 2018.

Aprobado: 22 de octubre del 2018. 
- ¿ ¿Hay una idea sobre la manera en que esa inmensa red de neuronas se organiza y funciona?

Abordemos primero la cuestión de la arquitectura de conjunto, que es una arquitectura de funcionamiento "en paralelo", lo que quiere decir que la información la tratan áreas múltiples de manera simultánea. Es el caso, por ejemplo, de los numerosos mapas comprometidos en la percepción visual, que al mismo tiempo tratan informaciones sobre los colores, las formas o los relieves, de los objetos observados.

Pero existe igualmente una organización jerárquica. Es una idea importante: yo sostengo una tesis, ya vieja, según la cual el encéfalo está estructurado en niveles de organización, que son otros tantos niveles de complejidad. Esos niveles de organización van de la molécula a la célula, de la célula a los circuitos elementales de neuronas, luego de estos a las redes y a organizaciones cada vez más globales; de alguna manera: iredes de redes!

A cada nivel de organización le corresponden funciones definidas. Una de las funciones asociadas a la célula es la propagación del influjo nervioso o la liberación de neurotransmisores; es pues una función de comunicación elemental. A nivel de los circuitos de neuronas, se puede hacer corresponder comportamientos más elaborados, pero aún muy simples, como el arco reflejo o programas de acciones fijas. Ahora bien, si uno se remonta a arquitecturas más complejas, se forman "asambleas de neuronas" cada vez más complejas. En el cerebro humano se van a codificar los conceptos, las representaciones simbólicas. Algunos territorios de nuestro encéfalo son más especializados en tal o cual tipo de representaciones. Finalmente, existe un nivel superior que se puede calificar como nivel de la razón, en el que se elaboran la organización de las conductas, la planificación de los comportamientos, las intenciones. Hay pues muchos niveles de organización que comprometen conjuntos de territorios distintos, pero en interacción. Las áreas implicadas en el pensamiento racional incluyen de manera privilegiada las áreas frontales y prefrontales que están muy desarrolladas en los primates, y especialmente en el hombre.

Esta manera de ver permite visualizar una arquitectura de conjunto, a la vez en paralelo y en niveles de organización jerárquica, con una intrincación profunda y una gran diversidad funcional.

El método que yo sugiero consiste pues en remontar de lo simple a lo complejo. El objetivo es poner en relación cada nivel de organización cerebral con un conjunto de funciones definidas, sabiendo que se establecen regulaciones entre niveles de abajo hacia arriba como de arriba hacia abajo. Esta puesta en correlación entre una red definida de neuronas, un conjunto de activida- 
des elementales y un comportamiento, la pudo llevar a cabo el neurobiólogo sueco Sten Grillner, a propósito de la forma de nadar de la lamprea, un pez muy arcaico.

Los comportamientos complejos solo pueden aparecer a partir de redes organizadas. Usted no puede dar cuenta de una conducta elaborada a partir de una sola célula o de una pequeña red de neuronas. Son propiedades emergentes que solo aparecen a un nivel de organización determinado.

- Si uno se remonta, como lo ha hecho, a niveles cada vez más complejos, ¿opera en ellos aún el método científico tradicional que aislaba funciones? ¿Se dispone de herramientas para aprehender esa complejidad creciente?

Hay muchos modos de exploración de las relaciones entre organización neuronal y funciones. El primero es el de la neuropsicología, disciplina médica que trata especialmente de describir las consecuencias de lesiones cerebrales definidas en el psiquismo y las conductas humanas. La neuropsicología nació en el siglo XIX con Paul Broca, él fue el primero en plantear la existencia de áreas especializadas que intervienen en el lenguaje. Este método fue utilizado más recientemente por François Lhermitte, por Tim Shallice y sus colegas. Ellos mostraron, por ejemplo, que algunas lesiones de la corteza frontal no alteran el lenguaje ni la memoria, pero afectan las capacidades de organización de las conductas. De este modo, una persona afectada de tales trastornos, en la cafetería no lograría organizar sus platos sobre la bandeja, aún cuando los reconozca perfectamente.

En mi libro Razón y placer (1997/1994) cito las observaciones del neurólogo ruso Alexandre Luria que relata el caso de un paciente con una lesión en el lóbulo frontal. Tiene dificultades para analizar el sentido del cuadro, aunque conoce todos sus elementos. Ante un cuadro del barón Klodt, "La última primavera" que representa a una muchacha moribunda sentada en un sillón, a quien sus padres miran tristemente, el individuo fija su atención en el vestido blanco de la joven y termina por confundir a la moribunda con una recién casada... Un caso más que demuestra que existe un territorio de la corteza cerebral responsable de la coordinación de las informaciones.

El segundo método de estudio consiste en utilizar las técnicas de imagenografía cerebral, como la cámara de positrones o la resonancia magnética funcional. Estos métodos permiten establecer mapas funcionales del sistema nervioso central y establecer los vínculos entre territorios cerebrales y funciones (visión, audición, reflexión, memoria...). Este método de imagenografía, con otros como la electroencefalografía o la magnetoencefalografía, pueden aportar mucho en los años venideros. 
- Pero estas apenas son técnicas que registran conexiones entre un área cerebral y una función. ¿Se ha explicado verdaderamente un mecanismo cuando se han encontrado las bases orgánicas de una función?

Me parece indispensable construir modelos formales para progresar en la comprensión de las funciones cerebrales. Un modelo es una arquitectura lógica, descrita bajo la forma de un programa de computador y que echa mano de "neuronas formales". Para dar cuenta de una función definida a un nivel de organización particular, si el modelo es adecuado debe "simular" una función. Es así como hemos podido construir con Stanislas Dehaene un modelo que logra superar la prueba de Wisconsin. Esa prueba consiste en pedirle a una persona que comprenda la regla de respuesta de una presentación de cartas para jugar, en función de la actitud positiva o negativa del examinador. Algunos pacientes que sufren de lesiones frontales fracasan en el examen. El modelo y la simulación informática permiten captar las operaciones movilizadas en el paso de la prueba. La organización de tales operaciones lógicas es lo que está perturbado en el paciente. Tenemos acá un ejemplo simple de tentativa de simulación de las funciones mentales superiores que puede ser útil a la vez, en la puesta en correspondencia estructura-función, y en el plano médico. Se han publicado muchos otros ejemplos en el curso de los últimos años, incluso sobre el acceso a la conciencia, siempre a cargo de Stanislas Dehaene y sus colaboradores.

- Algunos teóricos de las ciencias cognitivas consideran que se puede comprender el funcionamiento mental dejando de lado el estudio del estrato orgánico. Se trata de razonar a nivel de las estrategias de resolución de problemas aplicadas, independientemente de su pedestal material. ¿Qué piensa sobre esto?

El esquema clásico del cerebro-computador distingue la "quincallería", o lo "material", que aquí corresponde a las neuronas del sistema nervioso central, y el "programa" que se pone en la máquina, y que sería como describir los comportamientos bajo la forma de un programa de computador. Ahora bien, pienso que hay oportunidad para un enriquecimiento considerable de los conocimientos al estudiar las relaciones causales entre las funciones mentales y su soporte material -tal y como existe en el cerebro-, no solamente bajo la forma de un programa de computador abstracto. Las disociaciones funcionales provocadas por lesiones definidas permiten descubrir las operaciones que juegan en una función dada. Al comienzo, la tentación era globalizar.

"Disociar" funciones conduce a eliminar selectivamente algunas operaciones, al mismo tiempo que se conservan otras. Tomemos el caso de pacientes prosopagnósicos, es decir víctimas de una perturbación frente al reconocimiento de rostros. Se ha podido mostrar que algunas lesiones bilaterales del lóbulo temporal arrastran la prosopagnosia. Además, en las regiones homólogas en el 
mono, se puede aislar experimentalmente las células individuales que responden selectivamente a caras completas, incluyendo ojos, nariz y boca. Si se retiran algunas partes del rostro, esas neuronas ya no responden. Se puede mostrar que existen sistemas especializados en el reconocimiento de rostros que incluyen muchas etapas de complejidad creciente en este proceso. El proyecto de acá en adelante es partir de datos anatómicos, fisiológicos y comportamentales bien definidos para construir modelos "neuromiméticos" de funciones psicológicas particulares.

- A nivel de los procesos superiores, ¿no debería haber colaboración entre muchas disciplinas, la neurología que estudia las bases neuronales de las conductas, la psicología que estudia las conductas propiamente dichas sin buscar conocer sus bases biológicas?

Por supuesto que esta colaboración no solamente es deseable sino necesaria. No se pueden estudiar las bases neuronales de una función si no se ha estudiado la función en sí misma. Lo cual supone que los psicólogos hayan desarrollado ya sus propias teorías y modelos que le proponen luego a los neurobiólogos, y de igual manera recíprocamente. Es necesario que estas disciplinas hayan alcanzado un nivel de desarrollo suficiente para que converjan luego en la explicación. Las neurociencias cognitivas son ciencias federativas que reúnen a neurólogos, fisiólogos, psicólogos, lingüistas, antropólogos, filósofos.

- Su libro Razón y placer se dedica a un ambicioso programa: captar las raíces neuronales del placer estético.

En un texto de Razón y placer propuse una hipótesis para explicar las bases neuronales del placer estético. Claro que por lo demás, busqué ser muy prudente, solo se trata de propuestas y no de una teoría acabada. Pero en ese libro también se encuentran reflexiones sobre el proceder creativo, sobre las representaciones sociales y su propagación, sobre la historia de las ideas, etc. Asimismo, he querido mostrar cómo cada disciplina puede aportar su contribución a la comprensión del todo.

Deseo que ese libro muestre cómo es posible hacer frente a la colaboración entre neurociencias y ciencias humanas. Se trata de sugerir un método de investigación más que responder sobre el fondo a todas las preguntas que plantea la contemplación y la creación de una obra de arte.

- Esto va en contra de una posición que a menudo le es endilgada desde que publicó El hombre neuronal, esa de un reduccionista que querría explicar el funcionamiento mental a partir de su solo escalpelo...

Ese nunca ha sido mi punto de vista. En El hombre neuronal (1986) traté de puntualizar lo que se conocía de neurociencias en la época. Explicar todo el 
funcionamiento mental a partir del solo escalpelo me parece absurdo, lo que no quiere decir que no haya que usar el escalpelo. Mi propósito era simplemente decir: es importante sacar de los conocimientos actuales sobre el cerebro las consecuencias que arrojan para las otras ciencias. En muchos capítulos escribí explícitamente que se requería establecer conexiones entre neurociencias y ciencias del hombre, en particular la antropología. Es para mí una evidencia: no se puede aislar el cerebro del hombre de su entorno ni de su historia. En el cerebro del hombre se anudan de hecho tres evoluciones:

- una evolución biológica que va del mono al Homo sapiens;

- un desarrollo individual (la epigénesis) ligado a una conectividad singular, adquirida por estabilización selectiva y debida a la impronta del mundo exterior en el cerebro, y a la experiencia interior del individuo sobre sí mismo;

- la evolución de las culturas, que se ha hecho posible por las excepcionales capacidades de aprendizaje propias del cerebro humano.

Nunca he pretendido explicar al hombre a partir de la biología exclusivamente. No se puede comprender verdaderamente al hombre si no se tiene en cuenta estas tres evoluciones que comprometen la interacción del cerebro con su entorno. Siempre he dicho esto y por lo demás, he invertido muchas energías en trabajar a la vez en el plano teórico y experimental, en lo que se llama la "epigénesis", es decir, en las modulaciones de la organización de la red nerviosa que se desarrolla por la experiencia y por la actividad interna del organismo. Esta posición exclusivamente "reductora" que se me atribuye algunas veces, me es ajena; siempre he tratado de completarla con un recorrido sintético, de arriba abajo y por lo demás, igualmente materialista, pero que tiene en cuenta la vida individual, la interacción social y la evolución cultural.

\section{Referencias}

Changeux, J. P. (1986). El hombre neuronal. Madrid: Espasa-Calpe.

Changeux, J. P. (1997/1994). Razón y placer. Barcelona: Tusquets. 